\title{
Corrigendum: A pan-cancer proteomic perspective on The Cancer Genome Atlas
}

\author{
Rehan Akbani, Patrick Kwok Shing Ng, Henrica M.J. Werner, Maria Shahmoradgoli, Fan Zhang, Zhenlin Ju, \\ Wenbin Liu, Ji-Yeon Yang, Kosuke Yoshihara, Jun Li, Shiyun Ling, Elena G. Seviour, Prahlad T. Ram, John D. Minna, \\ Lixia Diao, Pan Tong, John V. Heymach, Steven M. Hill, Frank Dondelinger, Nicolas Städler, Lauren A. Byers, \\ Funda Meric-Bernstam, John N. Weinstein, Bradley M. Broom, Roeland G.W. Verhaak, Han Liang, \\ Sach Mukherjee, Yiling Lu \& Gordon B. Mills
}

Nature Communications 5:3887 doi: 10.1038/ncomms4887 (2014); Published 29 May 2014; Updated 28 Jan 2015

This Article contains an error in the Author contributions section that has resulted in incorrect credit for supervision of the network analysis. The correct Author contributions section is as follows:

R.A. and G.B.M. led the analysis in the paper. G.B.M. supervised the project and manuscript writing. R.A., P.K.S.N. and H.M.J.W. wrote much of the manuscript, supplement and response to reviewers. J.N.W. edited the manuscript and response to reviewers. M.S., G.B.M., P.K.S.N., Y.L., H.M.J.W., J.N.W., F.M.-B. and R.A. helped in interpreting the results. F.Z., Z.J., W.L., S.L. and J.-Y.Y. performed the analyses included in the paper and in the response to reviewers. K.Y. and R.G.W.V. performed purity analysis. J.L., H.L., B.M.B. and P.K.S.N. made the data and analyses available to the public at the TCPA portal and at the project home page. E.G.S. and P.T.R. performed function space analysis of the proteins. J.D.M., L.D., P.T., J.V.H. and L.A.B. provided unpublished lung cancer cell line data for validation. S.M.H., F.D. and N.S. performed network analyses under the supervision of S.M. and these authors contributed to writing parts of the manuscript. Y.L. generated the RPPA data under the supervision of G.B.M. 